\title{
Contrast-enhanced ultrasound for screening hepatocellular carcinoma: an implemented program at a semi-rural academic center
}

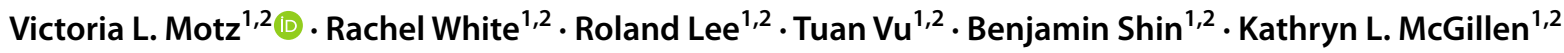

Received: 5 March 2021 / Revised: 13 April 2021 / Accepted: 21 April 2021 / Published online: 6 May 2021

(C) The Author(s), under exclusive licence to Springer Science+Business Media, LLC, part of Springer Nature 2021

\begin{abstract}
Purpose To describe our early experience using a contrast-enhanced ultrasound (CEUS) protocol for surveillance of hepatocellular carcinoma (HCC) at a semi-rural academic medical center.

Methods Retrospective, longitudinal study of the first 100 patients who underwent CEUS liver screening imaging over 2 years. Each patient underwent a standard of care abdominal ultrasound, which was checked with the radiologist, who searched for a focal lesion on the cine clips to target specifically with contrast. If none was present, the HCC contrast-enhanced screening protocol consisting of individual sweeps of the right and left lobes was performed from 0 to $60 \mathrm{~s}$ and 3-4 min post contrast—Lumason was utilized. Images, report details, and patient demographics were analyzed.

Results 66 men and 34 women (average age, $59 \pm 13$ years) were included. On average, the distance from patient's home to our institution was 39 miles (range 2-179 miles). The majority of our patients were covered under Private insurance (46\%) with $43 \%$ covered by Medicare. CEUS exams on average took $35 \mathrm{~min}$ to complete. Lumason was administered in split doses for an average total of $5 \mathrm{~mL}$ per exam. Of the 10 lesions identified, there were five LI-RADS 3, two LI-RADS 4, one LIRADS 5, two LI-RADS M, and one bland portal vein thrombus. There were no complications reported.

Conclusion This semi-rural single-center study demonstrates the feasibility of starting a HCC CEUS screening program. CEUS can be performed in conjunction with routine ultrasound imaging with added benefit of identifying and characterizing lesions in one setting.
\end{abstract}

Keywords Li-RADS $\cdot \mathrm{HCC} \cdot$ Ultrasound

Victoria L. Motz

Vmotz@pennstatehealth.psu.edu

Rachel White

Rwhite5@pennstatehealth.psu.edu

Roland Lee

Rlee6@pennstatehealth.psu.edu

Tuan Vu

Tuanvu@pennstatehealth.psu.edu

Benjamin Shin

Bshin1@pennstatehealth.psu.edu

Kathryn L. McGillen

Kmcgillen@pennstatehealth.psu.edu

Penn State College of Medicine, Hershey, PA, USA

2 Department of Radiology, Division of Abdominal Imaging, Penn State Health Milton S. Hershey Medical Center, Clinical Radiology Research Group, 500 University Dr, Hershey, PA 17033, USA

\section{Introduction}

Hepatocellular carcinoma (HCC) is the 4th most common cause of cancer-related mortality worldwide and the most common primary liver cancer [1]. Overall 5-year survival is estimated to be less than $12 \%$, which makes HCC the fastest rising cause of cancer-related death in the United States [2]. The development of HCC is highly associated with cirrhosis, and it is estimated that up to $95 \%$ of HCC patients have cirrhosis [3]. World health organizations have outlined various screening modalities in order to identify HCC lesions early, ultimately allowing for earlier treatment. Currently, the American Association for the Study of Liver Diseases (AASLD) guidelines recommends HCC screening to occur every 6 months in patients at risk for developing HCC [4]. The imaging modality of choice is abdominal ultrasound with or without alpha-fetoprotein (AFP) levels [4]. Ultrasound (US) as a HCC surveillance tool has a sensitivity of close to $80 \%$ and a specificity as high as 95\% [5]. Once HCC 
is suspected based on US imaging, staging and diagnosis confirmation are made with enhanced multiphasic computed tomography $(\mathrm{CT})$ or multiphasic magnetic resonance imaging (MRI) with contrast [6]. The use of CT or MRI has some disadvantages, namely use of contrast agents, which may be problematic in patients with impaired renal function, contrast allergies, and the radiation exposure incurred from multiphase CT. MRI also has contraindications, such as in patients with certain metallic foreign bodies or implants.

Contrast-enhanced ultrasound imaging (CEUS) is a dynamic, real-time imaging technique involving contrastenhanced images. When utilized in the liver, it has been used to identify and accurately characterize focal liver lesions. CEUS provides contrast-enhancement patterns of focal liver lesions without ionizing radiation and with improved temporal resolution compared with CT or MRI [7]. CEUS involves the intravenous injection of microbubble-based contrast agents known as ultrasound contrast agents (USCAs) consisting of tiny bubbles with a low-solubility gas core [8]. These gas-filled microbubbles act to highlight the intravascular space, but some may also be selectively phagocytized within the livers reticuloendothelial system, otherwise known as the Kupfer phase [9]. This Kupfer phase provides a post-vascular phase contrast enhancement of the liver parenchyma, highlighting focal liver lesions. USCAs are generally safe and are well tolerated in patients with renal failure. According to a large multi-center study of 23,188 patients who had been examined for liver lesions using USCAs, a serious adverse event rate of $0.0086 \%$ was reported, with no deaths [10]. Furthermore, a recent meta-analysis by Wu et al. assessed the use of CEUS in characterizing malignant from benign focal liver lesions with a sensitivity and specificity of $92 \%$ and $87 \%$, respectively [9].

The American College of Radiology has released the CEUS Liver Imaging Reporting and Data System (LIRADS) guidelines for the characterization of liver lesions concerning for HCC. The LI-RADS classification system uses the lesion size, type and degree of arterial phase enhancement, presence of washout, as well as the timing and degree of washout to categorize focal liver lesions in cirrhotic patients at risk of HCC. There are 6 classifications: LI-RADS $1-5$ and M. In a study by Terzi et al., CEUS LIRADS is reported to be highly specific for diagnosis of HCC without misdiagnosis of intrahepatic cholangiocarcinoma (ICC), which was previously thought to be a major limiting factor in CEUS's use in HCC screening [11]. CEUS is not currently recommended as a sole means of screening for HCC, but could be a valuable screening modality in the future.

The purpose of the present study is to describe our early experience with using dynamic contrast-enhanced ultrasound as standalone screening for hepatocellular carcinoma in cirrhotic patients in addition to routine grayscale ultrasound. Primary end points assessed the feasibility of performing this study at a 548-bed semi-rural academic medical center-was the study able to be performed in a typical ultrasound time slot and able to identify lesions as a screening test. At our institution, CEUS was initially utilized when a known cirrhotic patient at high risk for HCC was denied insurance coverage for multiphase CT or contrastenhanced MRI. Other indications include patients with MRI incompatible pacemaker/defibrillator, inability to fit in CT scanner due to body habitus, phobia prohibiting the safe use of CT or MRI, with relative contraindications of renal failure, or history of contrast reaction to CT or MRI contrast.

\section{Methods}

\section{Enrolled patients}

This is a retrospective longitudinal study describing our initial experience with CEUS for screening of HCC in high-risk patients at a 548-bed semi-rural medical transplant center. The study and data collection methods were approved by our Institutional Review Board. We reviewed 100 consecutive patients who received CEUS HCC screening protocol imaging from November 2018 to January 2020. Referral to this program was made from either hepatology $(85 \%)$, primary care $(7 \%)$, or transplant surgery $(6 \%)$. Indications for screening liver CEUS are listed in Table 1.

\section{CEUS HCC screening program protocol}

Patients first received a standard of care-grayscale and color Doppler ultrasound imaging of the abdomen. Cine clips were obtained through the liver. The images were then checked with the radiologist, who reviewed prior images for known focal or indeterminate lesions, and searched for a focal lesion on the cine clips, which would then be targeted specifically with contrast. If none was present, the HCC screening protocol was performed. If a focal lesion was first identified on grayscale, then it was characterized with contrast. During the study, our institution utilized Lumason with $2.5 \mathrm{~mL}$ dose injected through the IV on a Siemens S2000 ultrasound unit, followed by a $5-10 \mathrm{~mL}$ saline flush by the radiologist. The left lobe of the liver was usually imaged first as the patient was already in a supine position, which is usually adequate for left lobe visualization. As contrast enters the imaging field of view of the left lobe, the sonographer swept back and forth to image as much of the parenchyma as was sonographically visible, for $0-60 \mathrm{~s}$ to evaluate for any areas of enhancement or rapid washout. No imaging was performed from $1 \mathrm{~min}$ through $3 \mathrm{~min}$ as this time period does not contribute to LI-RADS diagnosis, reduces number of bubbles, and also to reduce "image fatigue." Sweeps through 
Table 1 Patient demographics

\begin{tabular}{|c|c|}
\hline \multicolumn{2}{|l|}{ Patient/study demographics } \\
\hline Characteristic & $\begin{array}{l}\text { Patients } \\
(N=100)\end{array}$ \\
\hline Age $($ mean \pm SD) & $59 \pm 13$ \\
\hline Gender $(\mathrm{M} / \mathrm{F})$ & $66 / 34$ \\
\hline \multicolumn{2}{|l|}{ Ethnicity } \\
\hline American Indian/Alaska Native & 0 \\
\hline Asian & 7 \\
\hline Native Hawaiian/other pacific islander & 0 \\
\hline Black or African American & 10 \\
\hline White/Caucasian & 69 \\
\hline Other & 2 \\
\hline Unknown & 12 \\
\hline Height & $171 \pm 10$ \\
\hline Weight & $91 \pm 24$ \\
\hline BMI & $31 \pm 7$ \\
\hline Distance to HMC & $39 \pm 34$ \\
\hline \multicolumn{2}{|l|}{ Insurance type } \\
\hline Medicare & 43 \\
\hline Private & 46 \\
\hline VA & 1 \\
\hline Medicaid & 10 \\
\hline \multicolumn{2}{|l|}{ Referral type } \\
\hline Gastroenterology/hepatology & 85 \\
\hline Primary care & 7 \\
\hline Transplant & 6 \\
\hline Other & 2 \\
\hline \multicolumn{2}{|l|}{ Cirrhosis etiology } \\
\hline Hepatitis B & 10 \\
\hline Hepatitis C & 18 \\
\hline NASH vs. NAFLD & 28 \\
\hline Alcohol & 15 \\
\hline Cardiogenic & 4 \\
\hline Cryptogenic & 3 \\
\hline Multiple/mixed type & 22 \\
\hline AFP baseline & $4.8 \pm 7.2$ \\
\hline MELDNa baseline & $12 \pm 7.5$ \\
\hline \multicolumn{2}{|l|}{ Reason for CEUS } \\
\hline HCC screen & 95 \\
\hline PV patency & 2 \\
\hline Screen for GB pathology & 2 \\
\hline RUQ pain & 1 \\
\hline Total time ultrasound & $35 \pm 11$ \\
\hline Total contrast dose $(\mathrm{mL})$ & 5 \\
\hline Number of contrast doses & 2 \\
\hline
\end{tabular}

the left lobe resume at $3 \mathrm{~min}$ and typically go through at least $4 \mathrm{~min}$ to evaluate for focal areas of delayed washout. Otherwise, any residual bubbles were burst, the patient was repositioned to best view the right lobe of the liver, and an additional dose of Lumason $2.5 \mathrm{~mL}$ is administered, with saline flush. The protocol was repeated through the right lobe. Attention was given to the portal vein enhancement during the right lobe imaging to ensure patency, and if not patent, to distinguish between bland and tumor thrombus.

\section{Image analysis}

The clinical radiology reports of the first 100 CEUS patients were reviewed as well as the raw images. The time elapsed between the time stamp from the first grayscale image and the last CEUS image was calculated to determine the total imaging time. The following characteristics were recorded: presence of lesion, size of lesion, LI-RADS score, number of contrast doses administered, total dose of contrast administered, visibility of lesion on grayscale versus CEUS imaging, and lesion enhancement. Any discrepancy in the data or reports was reviewed by the last author (K.M.), a boardcertified radiologist with 7 years of experience.

\section{Statistical analysis}

Descriptive statistical analyses were performed using IBM SPSS Statistics Version 26.0 (IBM Corporation, Armonk, NY, USA). Data on continuous variables are presented as mean \pm standard deviation. Data on categorical variables are expressed fractions (numerator/denominator) and percentages.

\section{Results}

Of the first 100 consecutive patients reviewed (mean age, 59 years \pm 13 years), 66 were male and 34 were female. The majority of our patient enrollment were White (69\% of participants) with the next highest ethnicity at $10 \%$, Black. Body mass index (BMI) was recorded because non-alcoholic fatty liver disease (NAFLD) was a major contributing factor to the development of cirrhosis in our region, with an average BMI of $31 \pm 7$ (normal range of 18.5-25 for adults). The majority of participants had cirrhosis secondary to NAFLD (34\% of study participants, 34/100). Table 1 details the distribution of liver disease etiology. CEUS imaging referral was strictly for HCC screening. Forty-three patients had baseline AFP levels at the time of CEUS, with an average AFP of $4.8 \pm 7.2$ (normal for non-pregnant adults is $<8.4$ ). Model for EndStage Liver Disease (MELDNa) was calculated to determine 3-month mortality in patients with cirrhosis and was calculated for $90 \%(90 / 100)$ of patients with average score of $12 \pm 7.5$. MELDNa scores $>9$ represent increased mortality risk. On average, the distance from the patient's home to our institution was 39 miles \pm 34 miles (range $2-179$ miles). The 
majority of our patients were covered under private insurance $(46 \%)$ with $43 \%$ covered by Medicare.

Of the 100 patients reviewed, 9 patients had imaging demonstrating LI-RADS lesions with one patient having two LI-RADS lesions (both were LI-RADS 3); all 10 LI-RADS lesions were characterized. 5/10 (50\%) were LI-RADS 3 lesions, 2/10 (20\%) LI-RADS 4, 1/10 (10\%) LI-RADS 5, and 2/10 (20\%) LI-RADS M. Taking into account that LIRADS 5 and $\mathrm{M}$ are malignancy equivalents, this patient population carried a $3 \%$ suspected malignancy rate $(3 / 100)$. By convention at our institution, benign lesions such as cysts and hemangiomas were not ascribed LI-RADS categorization by our radiologists, and so were not included in the "lesion" count or number of lesions characterized. LI-RADS lesion size on average was $1.8 \mathrm{~cm} \pm 0.9 \mathrm{~cm}$ (range of $0.8-3.2$ $\mathrm{cm})$. Of the 10 lesions, seven were located in the right lobe, three in the left lobe. One thrombus was identified in the portal vein, which did not enhance and was diagnosed as bland thrombus.

This study analyzed initial CEUS imaging from November 2018 to January 2020. The average time to perform each study was $35 \mathrm{~min} \pm 11 \mathrm{~min}$. Nearly all patients $(99 / 100)$ received two doses of contrast with an average total contrast volume of $5 \mathrm{~mL}$ of Lumason. Each lesion was also classified by its CEUS imaging characterization. Eight lesions were visualized on both grayscale standard imaging as well as CEUS imaging. Two lesions were seen on CEUS alone. CEUS characteristics of each lesion were recorded (Table 2). No complications were reported. On average, the rate of follow-up with additional imaging (CEUS, CT, or MRI) was $17 \%(17 / 100)$ with average time to follow-up imaging of 4.5 months \pm 4.9 .

\section{Discussion}

Our aim was to assess the feasibility of using CEUS for HCC screening in at-risk patients to augment the standard grayscale ultrasound currently being used. Our initial experience using CEUS as a screening tool for HCC in at-risk, cirrhotic patients showed that it is feasible to conduct this exam at a semi-rural medical center. We hypothesized that CEUS would be able to better characterize lesions in real time, offer fewer return visits for imaging by decreasing the need for follow-up CT or MRI imaging, and catch lesions that would otherwise be missed by standard grayscale ultrasound. Secondary endpoints evaluated safety of using Lumason contrast agent.

All of our 100 CEUS HCC screening sonographic examinations were completed within 46 min with an average time of $35 \mathrm{~min}$, not including time to place an IV. At our institution, CEUS time slots were allotted $1 \mathrm{~h}$ as our team was learning a new technology, additional images had to be
Table 2 Lesion demographics

\begin{tabular}{|c|c|}
\hline \multicolumn{2}{|l|}{ Lesions demographics } \\
\hline Characteristic & $\begin{array}{l}\text { Lesions } \\
(N=10)\end{array}$ \\
\hline \multicolumn{2}{|l|}{ Number of lesions } \\
\hline 0 & 91 \\
\hline 1 & 8 \\
\hline 2 & 1 \\
\hline \multicolumn{2}{|l|}{ Location of lesion } \\
\hline Right & 7 \\
\hline Left & 3 \\
\hline Right and left & 0 \\
\hline Caudate & 0 \\
\hline Lesion size & $1.8 \pm 0.9$ \\
\hline \multicolumn{2}{|l|}{ LI-RADS score } \\
\hline 1 and 2 & 0 \\
\hline 3 & 5 \\
\hline 4 & 2 \\
\hline 5 & 1 \\
\hline M & 2 \\
\hline \multicolumn{2}{|l|}{ Lesion visibility } \\
\hline Grayscale & 0 \\
\hline CEUS & 2 \\
\hline Grayscale and CEUS & 8 \\
\hline \multicolumn{2}{|l|}{ Lesion characteristic } \\
\hline Arterial hyperenhancement & 6 \\
\hline Slow wash out & 4 \\
\hline Fast washout & 3 \\
\hline Other & 1 \\
\hline
\end{tabular}

obtained, and each patient required an IV. Standard US time slots are $45 \mathrm{~min}$. Although, given more time and experience with CEUS, 45 min would be reasonable for performing CEUS. Standard CT timeslots at our institution are $20 \mathrm{~min}$, but do not include the time to place an IV, as we have a separate technologist whose sole purpose is to place IVs. MRI slots are similar to CEUS, at $45 \mathrm{~min}$, but also do not include time for IV placement. At our institution, a radiologist was present and administered each exam, which allowed for preliminary results at the conclusion of the exam, and the ability to answer questions. However, we understand that having a radiologist present for all CEUS exams may not be feasible at other institutions. CEUS's real-time evaluation also allowed radiologists to dynamically analyze parenchymal and tumor microperfusion at bedside, and at a more accurate temporal and spatial resolution when compared to CT and MRI [12]. The radiologist can also direct further imaging if needed, which could reduce callbacks or recommendation for additional imaging studies, whose importance increases at a semi-rural transplant center, where patients 
may travel long distances for a single study $(39 \pm 34$ miles in this study).

Our study revealed ten LI-RADS 3 or higher lesions among the first 100 patients (Table 2). Among the ten identified lesions, two lesions were seen on CEUS but not identified on grayscale ultrasound prior to contrast administration, although neither were proven malignant via subsequent CT.
Eight other lesions were seen on both grayscale and contrastenhanced imaging. Of these eight lesions, the LI-RADS 5 (one patient) and $\mathrm{M}$ lesions (two patients) were proven as malignant via four-phase $\mathrm{CT}$ and subsequently confirmed via transarterial chemoembolization therapy. The two lesions identified solely on post-contrast imaging, were not proven malignant, and they were relatively small $(0.8 \mathrm{~cm}$ and $2 \mathrm{~cm})$.
Fig. 1 CEUS shows a lesion (White Arrow) that enhances early, but is not seen on grayscale above background heterogeneity
Fig. 2 Same lesion with persistent enhancement in the periphery of segment 5 , beneath a small amount of ascites. Deemed a LI-RADS 3 on initial image interpretation, but could be a flash filling hemangioma or adenoma
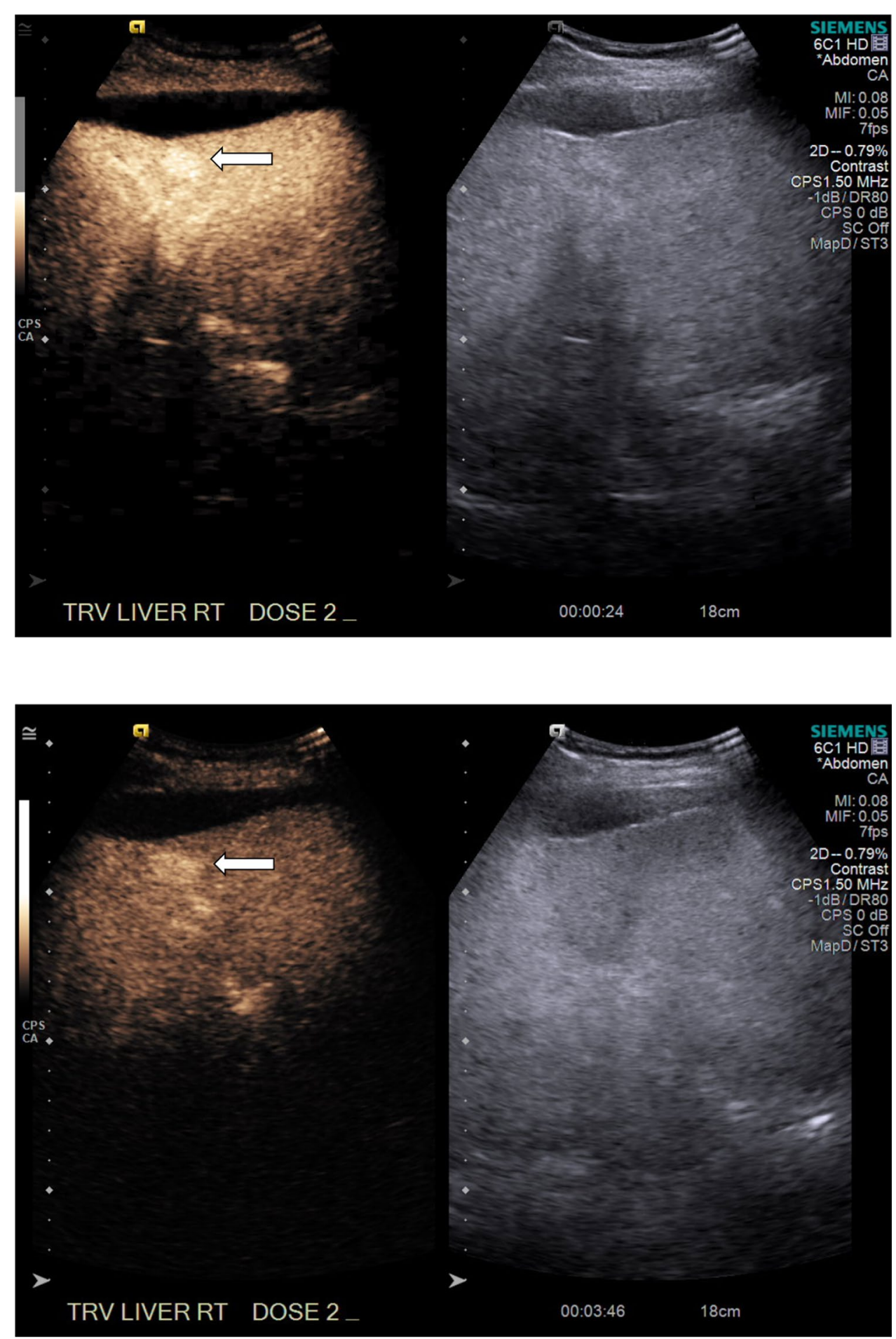
Fig. 3 MRI T2 shows a non-fat saturation and pre and post Eovist contrast of the lesions seen in Figs. 1 and 2. There is a perfusion anomaly present with geographic fatty deposition (not shown in its entirety). The lesion shows subtle nodular peripheral enhancement

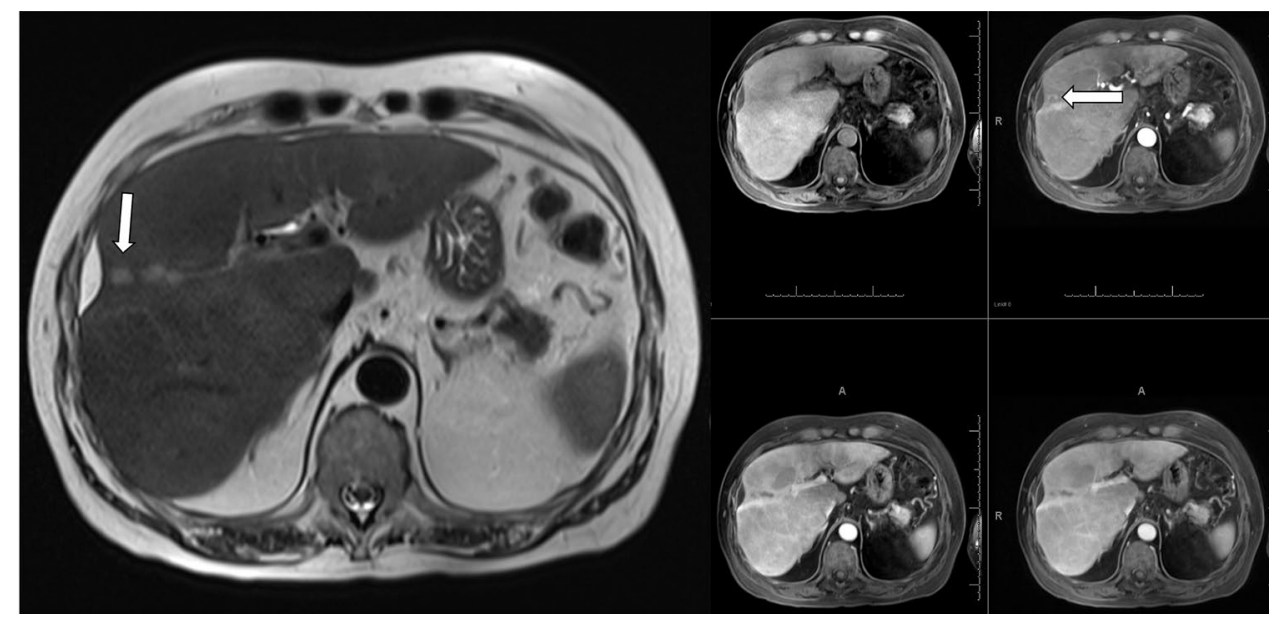

The first lesion was classified as LR-3 (Figs. 1, 2), which was later confirmed via MRI to be a hemangioma (Fig. 3). The second lesion was never followed up with additional imaging as this patient was lost to follow-up. Given these results, our study supports the findings of a similar study by Park et al. [13] showing minimal diagnostic value added to traditional grayscale imaging with CEUS if no lesion is first seen on grayscale imaging. However, due to small sample sizes of both studies, 100 patients in our study vs. 524 patients in Park et al. study, a larger study is required to confirm these findings.

The average age of patient population between these two studies are similar with our study at 59 and the Park et al. study at 54. However, our studies differed in type of contrast used, as well as range of ethnicity and cirrhosis etiology. While we identified positive lesions among $10 \%$ (10/100) of our participants with CEUS using Lumason, the Park et al. study investigated the utility of a different type of USCA with differing properties compared to those available in the U.S. as part of an HCC screening algorithm. Their study used Sonazoid (perfluorobutane)-enhanced US in HCC surveillance in patient with liver cirrhosis, which offers a 98\% Kupfer uptake as compared with the $7.3 \%$ seen with Lumason $[14,15]$. The Kupfer phase also lasts about $15 \mathrm{~min}$ with Sonazoid as compared to the 5 min with Lumason [15]. While Park et al. participants' ethnicity was homogenously Asian, our study included White (69\%), followed by Black (10\%) and Asian (7\%). Cirrhosis etiology was predominantly hepatitis B infection (94.1\%) in the Park et al. study, compared to ours, which had a wider range of etiology (Table 1) including NAFLD (28\%), Alcoholic (15\%), Multiple/Mixed type (22\%), Hepatitis C virus (18\%), with Hepatitis B virus less common (10\%).

The average size of our identified lesions was $1.8 \mathrm{~cm}$ with the smallest noted to be $0.8 \mathrm{~cm}$, illustrating the potential benefit of visualizing small liver lesions via the use of CEUS. In our study, malignant lesions (LR-5 and M) were all greater than $1 \mathrm{~cm}$ in size, although sub-centimeter lesions were present in lower categories. The literature supports improvement in visibility of typical characteristics of focal small liver lesions $(<1.5 \mathrm{~cm})$ with the use of contrast enhancement compared to grayscale standard imaging alone [16]. When compared to alternative modalities, typical HCC features are less frequently detected by using MRI in HCC lesions with diameters $<1.5 \mathrm{~cm}$ but more frequently observed using CEUS [12].

The complication rate for our study was $0 \%$ with no allergic reactions or adverse events noted. This supports prior literature that USCAs, specifically Lumason in our study, are very well tolerated and have been demonstrated to have a safe allergy profile. In 2005, a comprehensive review by the Contrast Media Safety Committee (CMSC) of the European Society of Urogenital Radiology (ESUR) demonstrated only $5 \%$ of patients exposed to US contrast agents experienced adverse events such as headache and nausea, which were all transient and self-limited [17]. In a more recent retrospective study consisting of 34,478 patients undergoing CEUS with SonoVue (European brand name for Lumason), only 40 cases $(0.12 \%)$ experienced adverse effects [18].

Among patients with LI-RAD positive results on their first CEUS screening in our study, the average follow-up time was 4.5 months ranging from 0 to 9 months after initial CEUS. As the majority of our screening CEUS cases were negative for lesion, many of our patients remained within "routine surveillance" guidelines, which at our institution, vary between 6 and 12 months in duration. According to literature, patients with CEUS LR-1 and LR-2 lesions are recommended to return to routine surveillance within 6 months; patients with CEUS LR-3 are recommended to obtain CT or MRI follow-up or repeat CEUS as an alternative within 6 months; patients with CEUS LR-4 are recommended for repeat CEUS or alternative diagnostic imaging within 3 months; patients with CEUS LR-5 and LR-M are recommended to follow multidisciplinary discussion for consensus 
management, usually accompanied by biopsy for $\mathrm{M}$ designations [19]. Our patient with a CEUS LR-5 lesion had a follow-up biopsy which confirmed the diagnosis. However, the majority of the other patients with positive results ranging from LR-3 to LR-4 on the initial CEUS screening did not adhere to follow-up guidelines as suggested by literature at the time of this study. At the time of analysis, followup imaging was available in only $17 \%$ of our patients. The average distance of 39 miles between patient homes and our medical center where the CEUS study was performed may play a potential role in the lack of follow-up adherence.

\section{Challenges on starting a CEUS HCC screening program}

There are several barriers on starting a contrast ultrasound service, including for HCC screening. These include the upfront cost of purchasing the software to allow for contrast use. Given COVID-19 and the financial burden it has placed on medical centers, it can be difficult to purchase the equipment necessary to perform CEUS. Other barriers include sonographer training as dynamic enhanced imaging is an entirely new concept. Once volume reaches a critical level, sonographers need to learn to place IV's, a new skill set that takes time to develop. Radiologists must also become familiar with LI-RADS for CEUS, which differs slightly from CT/ MRI LI-RADS, as well as the appearance of benign enhancing liver lesions, which can still occur in cirrhotic patients. In our practice, radiologists are in the room during the exam, which is a non-RVU generating activity. Furthermore, CPT codes for contrast ultrasound for the liver currently require a focal lesion, so if no lesion is identified, there is a revenue drop- the exam is charged as a routine liver ultrasound with the price of the contrast, which is significantly less than a contrast US liver charge.

\section{Limitations}

Limitations to screening HCC protocol include the following: small, sub-centimeter lesions may be missed on grayscale (noting that these lesions on LI-RADS for routine ultrasound are considered sub-threshold, US-2 and require short interval surveillance), and so may not receive their own dedicated contrast dose and could have transient, rapid enhancement that could be missed. The goal of the HCC protocol screen is that even if rapid enhancement is missed, if there is washout, it would be identified on the cine clips. Inherent weaknesses of ultrasound remain as limiting factors-the entirety of the liver may not be visible in large habitus patients, or may not be seen with standard CEUS software, that routinely images up to about $10 \mathrm{~cm}$ depth. The average BMI of our patients was 31 and ranged between 24 and 38 and approximately $1 / 3$ rd of our patients had cirrhosis related to NAFLD. Large body habitus and fat within the liver will inherently limit visualization via ultrasound, and CEUS is no exception.

Another limitation of this study is the small sample size, which was due in part to study design as this was a pilot study to investigate the feasibility of using CEUS as a screening tool for HCC in at-risk patient populations. However, in order for definitive conclusion to be drawn, a larger study comparing lesions seen on CEUS to CT/MRI or standard US is required.

\section{Future directions}

Further studies need to be performed to evaluate HCC screening via CEUS in at-risk populations. This includes both sensitivity and specificity compared to ultrasound as well as to CT/MRI for both negative studies and for lesions, and to evaluate for lesions seen exclusively on post-contrast imaging, to determine if contrast adds additional value over grayscale when the screening is negative.

\section{Conclusions}

A contrast ultrasound screening program can be a valuable addition to the different tools and modalities used by clinicians to screen high-risk patients for HCC. There is value added when able to get a screening grayscale ultrasound and a diagnosis for focal abnormality, malignant or benign, without having to return for a second imaging study, most commonly four-phase CT or MRI. It reduces burden on the system both monetarily for the patient and of their time, and it frees up more imaging slots for other patients. Further studies are needed to evaluate for the value added of contrast if no focal liver or portal vein abnormality is first seen on grayscale imaging.

Disclosures Victoria Motz, Rachel White, Roland Lee, Tuan Vu, Benjamin Shin, Kathryn McGillen: none

Funding None.

\section{References}

1. El-Serag HB, Rudolph KL. Hepatocellular carcinoma: epidemiology and molecular carcinogenesis. Gastroenterology. 2007;132:2557-2576.

2. Mittal S, El-Serag HB. Epidemiology of hepatocellular carcinoma: consider the population. J Clin Gastroenterol. 2013;47 Suppl:S2-6. 
3. Fattovich G, Stroffolini T, Zagni I, Donato F. Hepatocellular carcinoma in cirrhosis: incidence and risk factors. Gastroenterology. 2004;127:S35-50.

4. Marrero JA, Kulik LM, Sirlin CB, et al. Diagnosis, Staging, and Management of Hepatocellular Carcinoma: 2018 Practice Guidance by the American Association for the Study of Liver Diseases. Hepatology. 2018;68:723-750.

5. Singal A, Volk ML, Waljee A, et al. Meta-analysis: surveillance with ultrasound for early-stage hepatocellular carcinoma in patients with cirrhosis. Aliment Pharmacol Ther. 2009;30:37-47.

6. Heimbach JK, Kulik LM, Finn RS, et al. AASLD guidelines for the treatment of hepatocellular carcinoma. Hepatology. 2018;67:358-380.

7. Bartolotta TV, Vernuccio F, Taibbi A, Lagalla R. ContrastEnhanced Ultrasound in Focal Liver Lesions: Where Do We Stand? Semin in Ultrasound CT and MRI. 2016.

8. Jo PC, Jang HJ, Burns PN, Burak KW, Kim TK, Wilson SR. Integration of Contrast-enhanced US into a Multimodality Approach to Imaging of Nodules in a Cirrhotic Liver: How I Do It. Radiology. 2017.

9. Wu M, Li L, Wang J, et al. Contrast-enhanced US for characterization of focal liver lesions: a comprehensive meta-analysis. Eur Radiol. 2018;28:2077-2088.

10. Piscaglia F, Bolondi L, Italian Society for Ultrasound in M, Biology Study Group on Ultrasound Contrast A. The safety of Sonovue in abdominal applications: retrospective analysis of 23188 investigations. Ultrasound Med Biol. 2006;32:1369-1375.

11. Terzi E, Iavarone M, Pompili M, et al. Contrast ultrasound LIRADS LR-5 identifies hepatocellular carcinoma in cirrhosis in a multicenter restropective study of 1,006 nodules. $J$ Hepatol. 2018;68:485-492.
12. Schwarze VA-O, Marschner C, Völckers W, et al. Diagnostic value of contrast-enhanced ultrasound versus computed tomography for hepatocellular carcinoma: a retrospective, single-center evaluation of 234 patients. J Int Med Res. 2020;48:300060520930151.

13. Park JH, Park MS, Lee SJ, et al. Contrast-enhanced US with Perfluorobutane for Hepatocellular Carcinoma Surveillance: A Multicenter Diagnostic Trial (SCAN). Radiology. 2019;292:638-646.

14. Chung YA-O, Kim KA-OX. Contrast-enhanced ultrasonography: advance and current status in abdominal imaging.

15. Barr RG, Huang P, Luo Y, et al. Contrast-enhanced ultrasound imaging of the liver: a review of the clinical evidence for SonoVue and Sonazoid. Abdominal Radiology. 2020;45:3779-3788.

16. Yang W, Chen MH, Wu W, Dai Y, Fan ZH. Effects of Gray-Scale Ultrasonography Immediate Post-Contrast on Characterization of Focal Liver Lesions. Biomed Res Int. 2015;2015:193178.

17. Jakobsen JA, Oyen R, Thomsen HS, Morcos SK, Members of Contrast Media Safety Committee of European Society of Urogenital R. Safety of ultrasound contrast agents. Eur Radiol. 2005;15:941-945.

18. Hu C, Feng Y, Huang P, Jin J. Adverse reactions after the use of SonoVue contrast agent: Characteristics and nursing care experience. Medicine (Baltimore). 2019.

19. Bartolotta TA-OX, Terranova MC, Gagliardo C, Taibbi AA-OX. CEUS LI-RADS: a pictorial review. Insights Imaging. 2020;11:9.

Publisher's Note Springer Nature remains neutral with regard to jurisdictional claims in published maps and institutional affiliations. 